\title{
Successful Conservative Management of Hepatic Portal Venous Gas due to Anastomosis Leakage After a Sigmoidectomy
}

\author{
Injae Hong, Seong Woo Hong, Yeo Gu Chang, Byungmo Lee, Woo Yong Lee, Haeng Jin Ohe, Young Ki Kim \\ Department of Surgery, Inje University Seoul Paik Hospital, Seoul, Korea
}

In past decades, hepatic portal venous gas (HPVG) has rarely been reported, and the mortality rate has been very high. In most cases, surgical intervention was needed. Presently, abdominal computed tomography can be conveniently used to diagnose HPVG, which has various underlying causes and benign courses. We present the case of a patient with HPVG due to anastomosis leakage after a sigmoidectomy for diverticulitis; the patient was cured with conservative management.

Keywords: Hepatic portal venous gas; Sigmoid; Diverticulitis

\section{INTRODUCTION}

Hepatic portal venous gas (HPVG) is considered a rare and ominous condition, with a reported mortality rate of $75 \%$. Surgical intervention was needed in most cases in 1978 [1]. Currently, computed tomography (CT) can be used to diagnose patients with HPVG, which has diverse pathologies and minimal or absent clinical signs and symptoms [2]. Increasing numbers of patients with HPVG have been cured without surgery [3]. In a few reported cases, HPVG was associated with complications after gastrointestinal surgery [4-6]; nevertheless, we were unable to find any cases of HPVG associated with anastomotic leak after a colon resection. Here, we present a case of HPVG that was diagnosed after a sigmoidectomy for diverticulitis and managed successfully without surgery.

Received: October 30, 2017 - Accepted: March 23, 2018

Correspondence to: Seong Woo Hong, M.D.

Department of Surgery, Inje University Seoul Paik Hospital, 9 Mareunnae-ro, Jung-gu, Seoul 04551, Korea

Tel: +82-2-2270-0021, Fax: +82-2-2270-0247

E-mail: hongsw@paik.ac.kr

ORCID code: https://orcid.org/0000-0002-4691-5666

(C) 2019 The Korean Society of Coloproctology

This is an open-access article distributed under the terms of the Creative Commons Attribution NonCommercial License (http://creativecommons.org/licenses/by-nc/4.0) which permits unrestricted noncommercial use, distribution, and reproduction in any medium, provided the original work is properly cited.

\section{CASE REPORT}

A 29-year-old man was transferred to the Department of Surgery with an indication for surgery for recurrent sigmoid diverticulitis. He had experienced 2 attacks of sigmoid diverticulitis 10 months and 5 months before surgery. He was treated with oral antibiotics at an outpatient clinic. He was a hepatitis B antigen carrier, but the levels of liver enzyme were within normal limits. He had no other diseases. Antibiotics were administered for eight days immediately prior to surgery. At laparotomy, localized inflammation was seen at the sigmoid colon and the mesocolon. No sign of generalized peritonitis or walled-off abscess was noted. A sigmoidectomy and end-to-end anastomosis were performed. The pathology was acute suppurative to gangrenous diverticulitis with focal perforation.

Nine days after surgery, he had chills, and his body temperature was elevated to $39.2^{\circ} \mathrm{C}$. CT showed a focal wall defect at the colon anastomotic site and a tract-like long abscess connected to the defect (Fig. 1). Nil per os (NPO), fluid and antibiotic therapy resulted in immediate recovery. Ten days later, the patient started a soft diet and had bowel movements. Abdominal CT was repeated 16 days after the first CT to check the state of healing. CT showed that the focal wall defect of the anastomosis had disappeared, but minute air bubbles were detected in the mural portion (Fig. 2A). Unexpected findings included branch-like radiolucencies in the peripheral and the posterior portions of the lateral and the $\mathrm{S} 4 \mathrm{seg}$ ments of the liver, suggesting HPVG (Fig. 2B). He had no general 
Volume 35, Number 5, 2019

Ann Coloproctol 2019;35(5):282-284

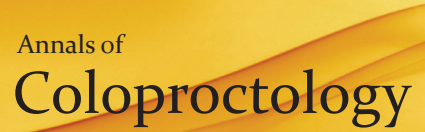

or abdominal symptoms. Body temperature was normal. No abdominal tenderness or rebound tenderness was detected. Blood parameters were as follows: white blood cell $4,950 / \mathrm{mm}^{3}$, C-reactive protein (CRP) $2.3 \mathrm{mg} / \mathrm{dL}$, and erythrocyte sedimentation rate (ESR) $56 \mathrm{~mm} / \mathrm{hr}$. CRP and ESR values were higher than normal, but showed a declining trend. Oral metronidazole was added to the previously used ciprofloxacin. The patient continued eating without any problem. No specific management step for HPVG was taken. Seven days after the second CT, a third CT was conducted to evaluate the HPVG. No HPVG was detected, and the sigmoidectomy site showed substantial improvement (Fig. 3). The

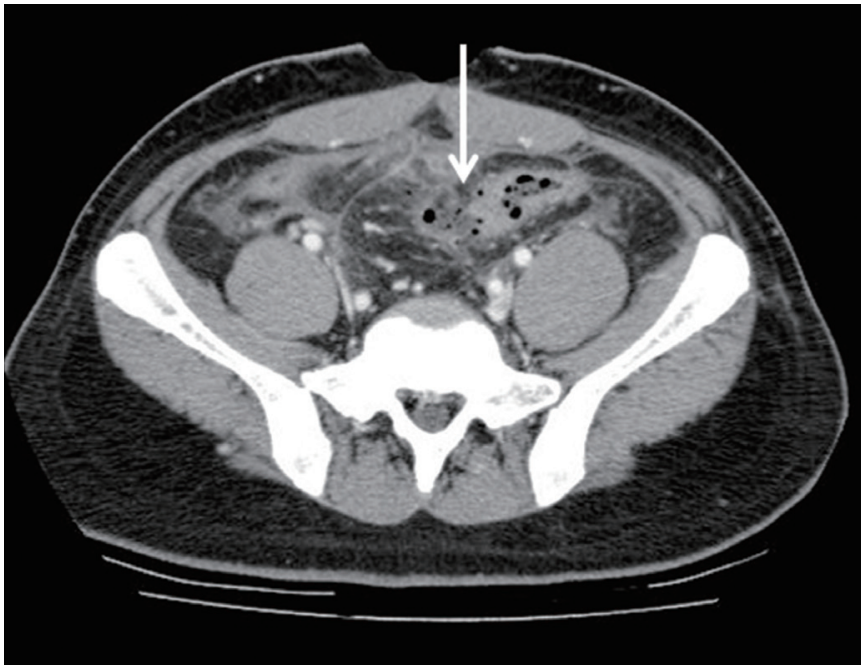

Fig. 1. A focal wall defect at the colon anastomosis site on the first computed tomography after surgery (white arrow).

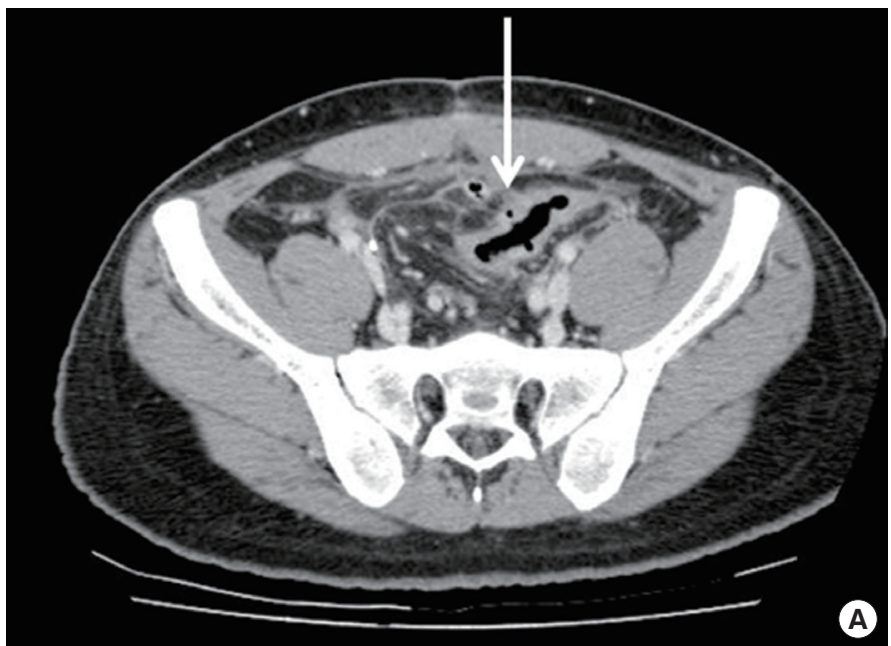

patient was discharged, and no symptoms related to HPVG or diverticulitis occurred during the three months following discharge. This study was approved by the Institutional Review of Board (IRB) of the Inje University Seoul Paik Hospital (PAIK 2018-10001) and the informed consent requirement was waived by IRB.

\section{DISCUSSION}

In 1955, Wolfe and Evans [7] first described HPVG in infants with necrotizing enterocolitis. Subsequently, it was reported in an increasing number of cases, including adults and pediatric pa-

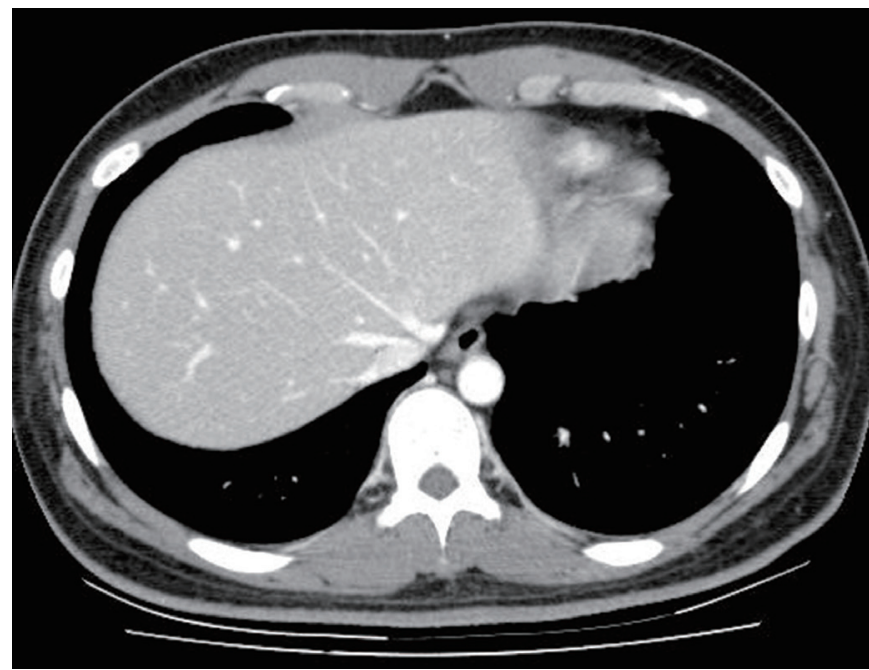

Fig. 3. Image from the third computed tomography (CT) done seven days after the second CT. The hepatic portal vein gas has disappeared.

Fig. 2. Images from the second computed tomography (CT), which was done 16 days after the first CT. (A) The focal wall defect of the anastomosis has disappeared, but minute air bubbles are seen in the mural portion (white arrow). (B) Unexpected findings (white arrow), which were considered to be due to hepatic portal venous gas, were seen as branches, like radiolucencies, in the peripheral and posterior portions of the lateral and the S4 segments in the liver. 
tients. The predisposing factors for HPVG include mucosal damage and bowel distension, which suggest increased intraluminal pressure and sepsis [1]. Our case illustrates mucosal tearing due to anastomosis leakage and sepsis due to an intra-abdominal abscess.

The diagnosis of HPVG is made based on branching radiolucencies within $2 \mathrm{~cm}$ of the liver capsule that are detected using plain radiography, CT, or ultrasonography. Pneumobilia is usually located in the central portion of liver more than $2 \mathrm{~cm}$ from the liver capsule [8]. In our case, CT showed intrahepatic branching radiolucencies at the peripheral portion of the liver. Recently, CT has been widely used to evaluate abdominal diseases or symptoms. Therefore, an increasing number of HPVG cases have been detected. The proportion of benign and non-life-threatening cases is increasing, too [8].

In 1978, Liebman et al. [1] reviewed 64 cases of HPVG in adults. In their report, the most common underlying causes were necrotic bowel (72\%), ulcerative colitis (8\%), intra-abdominal abscess (6\%), and bowel obstruction (3\%). In 2001, Kinoshita et al. [3] reviewed 182 cases of HPVG. The underlying diseases were bowel necrosis $(48.8 \%)$, digestive tract dilatation (13.0\%), abscess (12.3\%), ulcerative colitis (4.3\%), gastric ulcer (4.3\%), endoscopyrelated complication (4.3\%), Crohn disease (4.3\%), intraperitoneal tumor $(3.7 \%)$, cholangitis (1.9\%), pancreatitis (1.9\%), and hepatitis (1.2\%). A few patients with HPVG experienced complications after gastrointestinal surgery, including a gastrojejunal anastomosis [4], left hemicolectomy [5], and low anterior resection [6]. Unlike the 2 patients who had undergone a colectomy, the patient who had undergone a gastrojejunostomy experienced leakage at the anastomotic site. We were unable to find any cases in which HPVG was associated with anastomotic leakage after a colon resection.

The mortality rate for HPVG was $75 \%$ in 1978; thus, HPVG warranted urgent abdominal exploration in most instances [1]. At that time, most cases were associated with bowel ischemia and infarction, and early detection was impossible. In 2001, the mortality rate improved to $39 \%$, and conservative treatment was used in $49 \%$ of the cases with $39 \%$ mortality. Surgery was performed in $51 \%$ of the cases, and the mortality rate was 38\% [3]. HPVG with associated conditions, including digestive tract dilatation, gastric ulcer, ulcerative colitis, Crohn disease, and complications cause by the endoscopic procedure, was cured with conservative treatment [3]. Nelson et al. [9] suggested that the treatment for HPVG should be directed to the underlying disease. In patients with bowel necrosis or ischemia, an aggressive emergent laparotomy is recommended. In other cases, extensive investigation or conservative management, including NPO, hydration, antibiotics, and intervention drainage, should be considered [9].

Iannitti et al. [10] reviewed 26 patients with portal venous gas detected using CT. Fifteen patients were managed nonoperatively, and the survival rate was $60 \%$. They also concluded that portal venous gas on CT was associated with a wide range of pathologies that did not necessarily warrant surgical management. In our case, we detected HPVG following improvement in clinical symptoms and signs and managed it successfully with conservative treatment.

In conclusion, we report a case of HPVG associated with anastomotic leakage after a sigmoidectomy, which was cured with conservative treatment. We strongly suggest that careful conservative treatment can be applied to selected patients with HPVG.

\section{CONFLICT OF INTEREST}

No potential conflict of interest relevant to this article was reported.

\section{REFERENCES}

1. Liebman PR, Patten MT, Manny J, Benfield JR, Hechtman HB. Hepatic--portal venous gas in adults: etiology, pathophysiology and clinical significance. Ann Surg 1978;187:281-7.

2. Hou SK, Chern CH, How CK, Chen JD, Wang LM, Lee CH. Hepatic portal venous gas: clinical significance of computed tomography findings. Am J Emerg Med 2004;22:214-8.

3. Kinoshita H, Shinozaki M, Tanimura H, Umemoto Y, Sakaguchi S, Takifuji K, et al. Clinical features and management of hepatic portal venous gas: four case reports and cumulative review of the literature. Arch Surg 2001;136:1410-4.

4. Mognol P, Chosidow D, Marmuse JP. Hepatic portal gas due to gastro-jejunal anastomotic leak after laparoscopic gastric bypass. Obes Surg 2005;15:278-81.

5. Ginesu GC, Barmina M, Cossu ML, Feo CF, Fancellu A, Addis F, et al. Conservative approach to hepatic portal venous gas: a case report. Int J Surg Case Rep 2017;30:183-5.

6. Yamada T, Kan H, Matsumoto S, Machida T, Koizumi M, Shinji S, et al. A case of portal venous gas after rectal surgery without anastomotic leakage or bowel necrosis. J Nippon Med Sch 2015; 82:202-5.

7. Wolfe JN, Evans WA. Gas in the portal veins of the liver in infants; a roentgenographic demonstration with postmortem anatomical correlation. Am J Roentgenol Radium Ther Nucl Med 1955;74:486-8.

8. Abboud B, El Hachem J, Yazbeck T, Doumit C. Hepatic portal venous gas: physiopathology, etiology, prognosis and treatment. World J Gastroenterol 2009;15:3585-90.

9. Nelson AL, Millington TM, Sahani D, Chung RT, Bauer C, Hertl $\mathrm{M}$, et al. Hepatic portal venous gas: the $\mathrm{ABCs}$ of management. Arch Surg 2009;144:575-81.

10. Iannitti DA, Gregg SC, Mayo-Smith WW, Tomolonis RJ, Cioffi WG, Pricolo VE. Portal venous gas detected by computed tomography: is surgery imperative? Dig Surg 2003;20:306-15. 